\title{
Water-Tank Fish Algorithm: A New Metaheuristic for Optimization
}

\author{
Madhup Sukoon \\ Data Scientist
}

\author{
Haider Banka, $\mathrm{PhD}$ \\ Associate Professor, IIT Dhanbad
}

\begin{abstract}
This paper aims to introduce a new metaheuristic : The Water-Tank Fish Algorithm, modeled after the workings of the swim bladder in fish, to non-deterministically compute the optima for numerical optimization problems. To balance the explorative-exploitative behavior of a search, the proposed method uses a search localization routine which, after a general exploration, restricts the search to certain areas of the graph and intensifies it as the algorithm advances. The proposed method is tested over 40 benchmark mathematical functions and the results were found to be very encouraging.
\end{abstract}

\section{General Terms}

Metaheuristic, Nature Inspired, Optimization

\section{Keywords}

Fish, Buoyancy, Metaheuristic, Nature Inspired, Optimization

\section{INTRODUCTION}

The conventional approach to solving problems by computers is often found to be inadequate when solving real life problems, be it due to the multimodality of the problem, non-flexible construction or due to the sheer vastness of the search space. Such conditions render the conventional extensive search algorithms useless as the cost of finding the solution(s) in terms of CPU cycles and time, using such algorithms is very high. On the other hand, when similar problems are encountered in nature, the nature uses novel algorithms that are a result of several millennia of evolution. The algorithms used by nature are usually very efficient and outperform the algorithms designed by humans in terms of efficiency, accuracy and reliability. The algorithms used by nature are usually comprised of small agents that individually execute a very small and simple part of the algorithm, but when the output of each such agent is combined, the resulting output is often observed to be prominent. Natural algorithms are usually simple implementations of physical, chemical or mathematical rules and laws in nature and Nature Inspired Metaheuristics is a relatively new paradigm of Computer Science that aims at understanding and modeling such algorithms to create faster and more efficient ways for computers to solve real life problems. This paper presents a nature inspired algorithm, called the Water-Tank Fish Algorithm that simplifies and speeds up the task of optimizing mathematical functions. This algorithm is inspired from the working of the swim bladder in various marine animals. The swim bladder is an organ found in most of
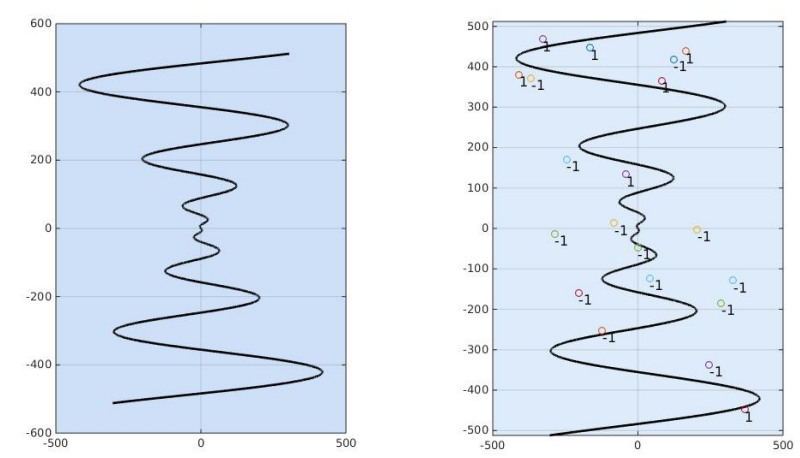

Fig. 1: Water Column. The vertical axis depicts the height of the Water Column as well as the input parameter of $f(x)$
Fig. 2: Water Column with 'fishes'. The number associated with each fish is its velocity. +1 is upwards and -1 is downwards.

the aquatic animals which allows them to change their altitude in water without much effort. The swim bladder is a basically a hollow pouch that contains air and allows the animal to change its size by contracting muscles, thus changing the density of its own body. Whenever there is a need for the animal to rise towards the surface, the swim bladder is allowed to expand, hence greatly reducing the density of the animal. Now, the buoyancy force acting on it pushes the animal upwards and the animal is able to change its altitude and go towards the surface with no or very little effort. Similarly, whenever there is a need for the animal to descend towards the base of the water body, the swim bladder is contracted, hence increasing the density of the animal's body and allowing gravity to bring it closer to the base of the water body with no or very less effort.

\section{WORKING}

For the sake of simplicity, the Schwefel's Function in 1 Dimension was considered as the function to be minimized. Thus, the fitness function $f(x)$ becomes:

$$
f(x)=-x \cdot \sin (\sqrt{|x|})
$$

We envision a vertical water column of unit density [Figure 1]. The height at each point in the water column corresponds to a value of the input parameter of $f(x)$. Next, intelligent objects, i.e. 'Fishes' were added in the Water-Tank at random 
heights[Figure 2], each fish having a unit mass. The 'Fishes' are intelligent because like real life fishes, they can change the upward(buoyant)/downward(weight) forces acting on them by changing their volume and thus attain an altitude of their choice in the water column. Each fish is given a unit velocity but the direction of the velocity (upwards or downwards) is randomized.

From here on, the algorithm runs independently. Each fish obtains its fitness value by evaluating $f(x)$ at its current height. Fitness value for each fish is calculated and the minimum and the maximum fitness values for each iteration are found out. Next, based on its own fitness value, the least and the greatest fitness values of the previous iteration and its current velocity, each fish changes its volume to a new value which gives the fish a new velocity. The algorithm is designed in such a way that whenever the fish goes from a better solution to a worse solution, its velocity increases. Also, whenever the fish approaches a good solution, its velocity decreases. This feature gives the algorithm a good control over the Eplorative-Exploitative behaviour. After attaining a new velocity, each fish travels with the new velocity for a fixed amount of time 'TransTime' (Translatoray Time) which is a tunable parameter.

\section{THEORY AND DERIVATIONS}

We know that the weight, or the downward force $\left(F_{w}\right)$ and the buoyant, or the upward force $\left(F_{b}\right)$ acting on an object of mass $m$ and volume $v$ immersed in a liquid of density $\rho$ are

$$
\begin{gathered}
F_{w}=m \cdot g \\
F_{b}=v \cdot \rho \cdot g
\end{gathered}
$$

where $g$ is the gravitational acceleration. The net force acting on such an object would be the resultant of the gravitational and the buoyant forces. Since the two forces act in opposite directions, the resultant can be calculated by subtracting them.

$$
F=F_{b}-F_{w}
$$

The net force on an object can also be denoted as the product of its mass $m$ and its acceleration $a$. Therefore,

$$
m \cdot a=v \cdot \rho \cdot g-m \cdot g
$$

For an object of unit mass submerged in a fluid of unit density, the equation becomes

$$
a=(v-1) \cdot g
$$

Assuming this object was subjected to this acceleration for time $t$ and multiplying both sides by $t$, the following equation comes forth:

$$
\begin{gathered}
a \cdot t=(v-1) \cdot g \cdot t \\
\text { NewVelocity }=\text { CurrentVelocity }+a \cdot t
\end{gathered}
$$

Using this equation in the context of the algorithm, for each fish of unit mass in a column of water of unit density, the following equation can be used to update the velocity of the fish.

$$
\text { NewVelocity }=\text { CurrentVelocity }+(v-1) \cdot g \cdot t
$$

The term $g \cdot t$ is dimensionally equal to velocity. $g$ is a constant, and the algorithm is constructed to adjust the value of $t$ such that $g \cdot t$ becomes equal to the current velocity of the object. Hence,

NewVelocity $=$ CurrentVelocity $+(v-1) \cdot$ CurrentVelocity

$$
\Rightarrow \text { NewVelocity }=\text { CurrentVelocity } \cdot v
$$

The volume of the fish is an indicator of the fitness of the solution being represented by the current location of the fish. The fish should slow down as it approaches a good solution to enable the exploitation of that solution. Also, the velocity of a fish should increase when it approaches a bad solution so that it can be skipped quickly. Therefore, a relation between the fitness of a fish's location and the volume of that fish is established in such a way that in a given iteration, the fittest fish will have a volume of 0 and the most unfit fish will have a volume of 1 . This can be further scaled by multiplying it with any scalar value $x$ which will multiply the velocity of the least fit fish by $x$ and set the velocity of the fittest fish as 0 while scaling the velocity of all other fishes in this range. To do this, the volume $v$ of a fish $j$ in the iteration $i$ is defined as:

$$
v_{j i}=x \cdot \frac{\text { maxfit }_{i}-\text { FitnessFunction }_{\left(\text {Position }_{j}\right)}}{\text { maxfit }_{i}-\text { minfit }_{i}}
$$

and arrive at the equation to update the velocity of a given fish $j$ in a given iteration $i$ :

$$
V_{j i}=U_{j i} \cdot x \cdot \frac{\text { maxfit }_{i}-\text { FitnessFunction }_{\left(\text {Position }_{j}\right)}}{\text { maxfit }_{i}-\text { minfit }_{i}}
$$

where $x$ is the scaling factor, $V_{j i}$ and $U_{j i}$ are respectively the new and current velocities of the fish $j$ and $\max f_{i t}$ and $\operatorname{minf}_{i} i_{i}$ are the outputs of the fitness function of the fittest and the least fit fish respectively for a given iteration $i$.

\section{ALGORITHM AND PARAMETERS}

Following are the components and parameters that build up the algorithm:

$n$ is the number of fishes/candidates used per dimension, e.g. using a value of $n=25$ would cause the algorithm to run with 50 candidates when evaluating a function in two dimensions.

FitnessFunction() is the function that returns the relative fitness, or goodness of a solution for the function to be optimized. For example, in case of finding the maxima of a function, the fitness function would be the same as the function to be optimized, whereas to find the minima of a function, the fitness function would be the reciprocal of the function to be optimized.

position of a candidate refers to the solution being represented by that candidate.

$k$ is a tunable parameter that determines the minimum non zero velocity at which a fish can travel. Smaller the value of $k$, greater is the search intensity in the exploitative phase. However, very small values of $k$ will slow down the convergence of the algorithm. Good results were obtained for $k \leftarrow 0.1$

TransTime corresponds to the Step Size for the algorithm. Very small values will slow down the convergence whereas large values may result in optimas getting skipped.

$D_{\max }, D_{\min }$ are respectively the maximum and minimum values allowed for a given dimension. Any candidate going beyond these values is reset to a valid position.

$x$ is the scaling factor which determines by how many times the velocity of each candidate increases or decreases. For example, setting the value of 2 would cause the velocity of the least fit candidate to double.

The proposed algorithm is as follows: 
Initialization:: Create a set of $\mathrm{N}$ randomly selected candidates (fishes) and assign each one a unit velocity $V$ in a random direction.

foreach $j \in N$ do

| Fitness $s_{j} \leftarrow$ FitnessFunction $\left(\right.$ Position $_{j}$ )

end

foreach iteration $i$ do

$\operatorname{maxfit}_{i} \leftarrow \max \left(\right.$ Fitness $_{1}$, Fitness $_{2} \ldots$ Fitness $\left._{N}\right)$

minfit $_{i} \leftarrow \min \left(\right.$ Fitness $_{1}$, Fitness $_{2} \ldots$ Fitness $\left._{N}\right)$

foreach $j \in N$ do

$V_{j} \leftarrow V_{j} \cdot x \cdot \frac{\text { maxfit }_{i}-{\text { FitnessFunction }\left(\text { Position }_{j}\right)}_{\text {maxfit }_{i}-\text { minfit }_{i}}}{\text { max }}$

if $\left(V_{j} \neq 0\right)$ and $\left(\left|V_{j}\right|<k\right)$ then

$\mid V_{j} \leftarrow k \cdot \hat{V}_{j}$

end

foreach dimension $D$ do

Position $_{j_{D}} \leftarrow$ Position $_{j_{D}}+V_{j_{D}} \cdot$ TransTime

if $\left(\right.$ Position $\left._{j_{D}}>D_{\text {max }}\right)$ or $\left(\right.$ Position $\left._{j_{D}}<D_{\text {min }}\right)$

then

end

Position $_{j_{D}} \leftarrow \operatorname{Random}\left(D_{\text {max }}, D_{\text {min }}\right)$

end

Fitness $_{j} \leftarrow$ FitnessFunction $\left(\right.$ Position $\left._{j}\right)$

end

if $\max _{i t_{i}}<\max \left(\right.$ Fitness $_{1}$, Fitness $_{2} \ldots$ Fitness $\left._{N}\right)$ then

foreach $j \in N$ do

if Fitness $_{j}==\max$ fit $_{i}$ then

${ }_{\text {end }} V_{j} \leftarrow k \cdot$ RandomV $\hat{V}$ locity

end

end

end

Algorithm 1: The Water-Tank Fish Algorithm

\section{REUSLT AND CONCLUSION}

The Algorithm was found to be working well for over 30 tested benchmark functions and the results were found quite encouraging. The results for these tests using the general hyperparameters $n=25, x=10$,TransTime $=1, k=0.5$ and 1000 iterations are present in [Table 1]. The selected hyperparameters resulted in quick convergence of few functions, while non-convergence of some others. Further research into enhancement of the algorithm and improving the parameters may result in faster and better convergence for optimization problems.

\section{REFERENCES}

[1] Berat Doğan and Tamer Ölmez. A new metaheuristic for numerical function optimization: Vortex search algorithm. Information Sciences, 293:125-145, 2015.

[2] James Kennedy. Particle swarm optimization. In Encyclopedia of machine learning, pages 760-766. Springer, 2011.

[3] Esmat Rashedi, Hossein Nezamabadi-Pour, and Saeid Saryazdi. Gsa: a gravitational search algorithm. Information sciences, 179(13):2232-2248, 2009.

[4] S Salcedo-Sanz, J Del Ser, I Landa-Torres, S Gil-López, and JA Portilla-Figueras. The coral reefs optimization algorithm: a novel metaheuristic for efficiently solving optimization problems. The Scientific World Journal, 2014, 2014.
[5] Sait Ali Uymaz, Gulay Tezel, and Esra Yel. Artificial algae algorithm (aaa) for nonlinear global optimization. Applied Soft Computing, 31:153-171, 2015.

[6] Xin-She Yang. A new metaheuristic bat-inspired algorithm. In Nature inspired cooperative strategies for optimization (NICSO 2010), pages 65-74. Springer, 2010. 
Table 1. : 1000 Iterations of the algorithm with the hyperparameters $n=25, x=10$, TransTime $=1, k=0.5$

\begin{tabular}{|c|c|c|c|c|c|}
\hline Function & Dimensions & $\begin{array}{l}\text { Actual Solu- } \\
\text { tion }\end{array}$ & Actual Value & Achieved Solution & Achieved Value \\
\hline Ackley & 1 & 0 & 0 & -0.0041227611 & 0.0173960556107257 \\
\hline Ackley & 2 & 0,0 & 0 & $-0.038455785,0.068230381$ & 0.378020967538511 \\
\hline Ackley & 3 & $0,0,0$ & 0 & $\begin{array}{ll}0.30507709, & -0.076191935, \\
-0.43843236 & \end{array}$ & 3.04457159142419 \\
\hline Beale & 2 & $3 ., 0.5$ & 0 & $3.0571899,0.51424233$ & 0.000495481893369319 \\
\hline Booth & 2 & $1 ., 3$. & 0 & $1.0163313,2.9883048$ & 0.000489463201684794 \\
\hline BukinN6 & 2 & $-10 ., 1$ & 0 & $-6.9029627,3.4977471 e-06$ & 0.217993018112742 \\
\hline DeJongsF1 & 1 & 1 & 0 & 0.00020047047 & $4.01884104549635 E-08$ \\
\hline DeJongsF1 & 2 & 1,1 & 0 & $0.026106869,-0.0039498456$ & 0.000697169886649546 \\
\hline DeJongsF1 & 3 & $1,1,1$ & 0 & $\begin{array}{l}0.023770792,-2.0764046 e-05 \\
-0.062884812\end{array}$ & 0.00451955064011924 \\
\hline DeJongsF2 & 2 & 1,1 & 0 & $0.98409745,0.9709178$ & 0.00086297836283582 \\
\hline DeJongsF2 & 3 & $1,1,1$ & 0 & $1.067836,1.2363416,1.5516065$ & 1.03656871279967 \\
\hline DeJongsF3 & 1 & -5.12 & 0 & -5.1178172 & 0 \\
\hline DeJongsF3 & 2 & $\begin{array}{l}-5.12 \\
-5.12\end{array}$ & 0 & $-5.0329518,-5.102256$ & 0 \\
\hline DeJongsF3 & 3 & $\begin{array}{l}-5.12 \\
-5.12 \\
-5.12\end{array}$ & 0 & $\begin{array}{ll}-5.057755, & -4.738543, \\
-5.0960696 & \end{array}$ & 1 \\
\hline DeJongsF4 & 1 & 0 . & 0.528742973100137 & -0.30920123 & -1.33513214062436 \\
\hline DeJongsF4 & 2 & $0 ., 0$. & -1.27291194215612 & $-0.71223362,0.43683808$ & -1.88493587494313 \\
\hline DeJongsF4 & 3 & $0 ., 0 ., 0$. & -1.43273398094344 & $\begin{array}{ll}0.15195432, & 0.21170982, \\
-0.80820223 & \\
\end{array}$ & -2.96160371470233 \\
\hline DeJongsF5 & 2 & $\begin{array}{l}-32.32 \\
-32.32\end{array}$ & 1 & $-32.053942,-31.892482$ & $1.56953055398801 E-06$ \\
\hline DeJongsF5 & 3 & $\begin{array}{l}-32.32 \\
-32.32 \\
-32.32\end{array}$ & 1 & $\begin{array}{ll}-31.855933, & -31.77467 \\
54.413737 & \end{array}$ & 0.000139833945166297 \\
\hline Easom & 2 & $\begin{array}{l}3.14159265 \\
3.14159265\end{array}$ & -1 & $2.9583695,3.1051145$ & -0.948904646806459 \\
\hline Eggholder & 2 & $\begin{array}{l}512 ., \\
404.2319\end{array}$ & -959.6407 & $480.97693,431.51503$ & -956.562077488447 \\
\hline Ellipsoid & 2 & $0 ., 0$. & 0 & $0.1676686,0.0011329992$ & 1.31179989211617 \\
\hline Ellipsoid & 3 & $0 ., 0 ., 0$. & 0 & $\begin{array}{ll}-2.1879088, & -0.10190603, \\
0.00016676634 & \\
\end{array}$ & 15.1995941911551 \\
\hline FiveWellPotential & 2 & $4.92,-9.89$ & -1.4616 & $-4.4899612,-9.9246877$ & -1.46325053815003 \\
\hline GoldsteinPrice & 2 & $0 .,-1$. & 3 & $-0.0066038267,-0.99761103$ & 3.01670837214381 \\
\hline Griewank & 1 & 0. & 0 & 0.010231296 & -0.999947634581868 \\
\hline Griewank & 2 & $0 ., 0$. & 0 & $0.0074102142,0.30486179$ & -0.976804593186793 \\
\hline Griewank & 3 & $0 ., 0 ., 0$. & 0 & $\begin{array}{ll}28.66145, & -8.9204369, \\
-6.4127396 & \\
\end{array}$ & -0.548384003356077 \\
\hline HyperEllipsodic & 1 & 0. & 0 & 0.0003950471 & $1.56062213969636 E-07$ \\
\hline HyperEllipsodic & 2 & $0 ., 0$. & 0 & $-0.027227885,0.0021958663$ & 0.000751001404451596 \\
\hline HyperEllipsodic & 3 & $0 ., 0 ., 0$. & 0 & $\begin{array}{ll}0.18424519, & 0.02916251, \\
-0.067178746 & \\
\end{array}$ & 0.0491861461738183 \\
\hline
\end{tabular}


Table 2. : Contd.: 1000 Iterations of the algorithm with the hyperparameters $n=25, x=10$, TransTime $=1, k=0.5$

\begin{tabular}{|c|c|c|c|c|c|}
\hline Function & Dimensions & $\begin{array}{l}\text { Actual Solu- } \\
\text { tion }\end{array}$ & Actual Value & Achieved Solution & Achieved Value \\
\hline KTablet & 1 & 0 . & 0 & $-2.7289275 e-05$ & $7.44704544989876 E-06$ \\
\hline KTablet & 2 & $0 ., 0$. & 0 & $0.01962894,-0.047150401$ & 26.0845558155429 \\
\hline KTablet & 3 & $0 ., 0 ., 0$. & 0 & $\begin{array}{ll}-0.10278668, & 0.13527152, \\
-0.040414348 & \end{array}$ & 304.968049188932 \\
\hline LeviN13 & 2 & $1 ., 1$. & 0 & $1.0075451,0.98653553$ & 0.00528870401921487 \\
\hline Matyas & 2 & $0 ., 0$. & 0 & $0.0049347138,0.022676404$ & $8.63156348112673 E-05$ \\
\hline McCormick & 2 & $\begin{array}{l}-0.54719 \\
-1.54719\end{array}$ & -1.9133 & $-0.54840606,-1.5419619$ & -1.91317439925728 \\
\hline Michalewicz & 1 & 0. & -1.8013 & 2.2029037 & -0.801303410044765 \\
\hline Michalewicz & 2 & $0 ., 0$. & -1.8013 & $2.1981633,1.5796817$ & -1.79772835592603 \\
\hline Michalewicz & 3 & $0 ., 0 ., 0$. & -1.8013 & $2.2046798,1.550115,1.3266738$ & -2.643960498653 \\
\hline Perm & 1 & 1. & 0 & 0.99933761 & $1.75503152427629 E-06$ \\
\hline Perm & 2 & $1 ., 0.5$ & 0 & $0.406144,0.89923009$ & 0.000132629882782494 \\
\hline Perm & 3 & $\begin{array}{lr}1 ., & 0.5, \\
0.33333333\end{array}$ & 0 & $\begin{array}{ll}0.98623339, & 0.50819539, \\
0.34634396 & \end{array}$ & 0.00443758260776505 \\
\hline Rastrigin & 1 & 0 . & 0 & 0.00021282932 & $8.98643010506777 E-06$ \\
\hline Rastrigin & 2 & $0 ., 0$. & 0 & $-0.0069979604,0.0017523451$ & 0.010323190049673 \\
\hline Rastrigin & 3 & $0 ., 0 ., 0$. & 0 & $\begin{array}{ll}0.002305865, & -1.0046031, \\
-1.0246121 & \end{array}$ & 2.18362787257742 \\
\hline Rosenbrock & 2 & 1,1 & 0 & $1.0731988,1.1482807$ & 0.0065655954547213 \\
\hline Rosenbrock & 3 & $1,1,1$ & 0 & $\begin{array}{ll}0.83562784, & 0.76274611, \\
0.6346623 & \\
\end{array}$ & 0.778610998763602 \\
\hline SchafferN2 & 2 & $0 ., 0$. & 0 & $-0.046018115,-0.22775235$ & 0.00252708305809375 \\
\hline SchafferN4 & 2 & $0 ., 1.25313$ & 0 & $-1.4193211,0.71794606$ & 0.295307238796496 \\
\hline Schwefel & 1 & 420.9687 & 0 & 420.97716 & $2.16514255998168 E-05$ \\
\hline Schwefel & 2 & $\begin{array}{l}420.9687 \\
420.9687\end{array}$ & 0 & $422.54418,420.74158$ & 0.319875263381392 \\
\hline Schwefel & 3 & $\begin{array}{l}420.9687 \\
420.9687 \\
420.9687\end{array}$ & 0 & $\begin{array}{ll}425.31271, & 419.62471, \\
424.77234 & \end{array}$ & 4.43754593819933 \\
\hline Shuberts & 2 & $0 ., 0$. & -186.7309 & $821.66643,-333.81308$ & -186.62074289197 \\
\hline SixHumpCamel & 2 & $\begin{array}{l}-0.0898, \\
0.7126\end{array}$ & -1.0316 & $1.2459454,-0.7818169$ & 0.0624144850676566 \\
\hline Sphere & 1 & 1 & 0 & $-1.778093 e-05$ & $3.16161467982839 E-10$ \\
\hline Sphere & 2 & 1,1 & 0 & $-0.005790638,0.0029694072$ & $4.23488678610381 E-05$ \\
\hline Sphere & 3 & $1,1,1$ & 0 & $\begin{array}{ll}0.13125962, & -0.015673727, \\
0.13781636 & \\
\end{array}$ & 0.0364681025243178 \\
\hline StyblinskiTang & 1 & -2.903534 & -39.166165 & -2.9035779 & -39.1661656704192 \\
\hline StyblinskiTang & 2 & $\begin{array}{l}-2.903534 \\
-2.903534\end{array}$ & -78.33233 & $-2.8969658,-2.9074079$ & -78.3313272406072 \\
\hline StyblinskiTang & 3 & $\begin{array}{l}-2.903534 \\
-2.903534 \\
-2.903534\end{array}$ & -117.498495 & $\begin{array}{ll}-2.9031452, & -2.9732501, \\
-2.853447 & \end{array}$ & -117.36981973155 \\
\hline SumOfDifferentPower & 1 & 0. & 0 & $8.4117504 e-05$ & $7.07575447890102 E-09$ \\
\hline SumOfDifferentPower & 2 & $0 ., 0$. & 0 & $0.0023639078,-0.0029248747$ & $5.61308204456128 E-06$ \\
\hline SumOfDifferentPower & 3 & $0 ., 0 ., 0$. & 0 & $\begin{array}{ll}-0.0026558266, & 0.025841037, \\
-0.030436675 & \\
\end{array}$ & $2.51672057430445 E-05$ \\
\hline ThreeHumpCamel & 2 & $0 ., 0$. & 0 & $0.013057004,0.0097548151$ & 0.000563465279028625 \\
\hline WeightedSphere & 1 & 0. & 0 & $6.2633257 e-05$ & $3.92292489471659 E-09$ \\
\hline WeightedSphere & 2 & $0 ., 0$. & 0 & $-0.0081266527,0.017223001$ & 0.00065930599773335 \\
\hline WeightedSphere & 3 & $0 ., 0 ., 0$. & 0 & $\begin{array}{l}0.11946413 \\
-0.05483658\end{array}$ & 0.033688788036058 \\
\hline XinSheYang & 1 & 0. & 0 & -0.00011601051 & 0.00011601051198272 \\
\hline XinSheYang & 2 & $0 ., 0$. & 0 & $-0.03294053,-0.003498247$ & 0.0363988141133762 \\
\hline XinSheYang & 3 & $0 ., 0 ., 0$. & 0 & $\begin{array}{l}-1.171776, \\
1.1780393\end{array}$ & 0.186041116065492 \\
\hline Zakharov & 1 & 0. & 0 & -0.00011924896 & $1.77753940659359 E-08$ \\
\hline Zakharov & 2 & $0 ., 0$. & 0 & $-0.012220399,0.018454652$ & 0.00064232107700958 \\
\hline Zakharov & 3 & $0 ., 0 ., 0$. & 0 & $\begin{array}{l}-0.11657572 \\
-0.13483264\end{array}$ & 0.0834578370437009 \\
\hline
\end{tabular}

